\section{Two of a kind}

Cytoskeletal proteins regulate cell adhesion and motility, and some have even been shown to mediate cell survival. In the June issue of Cancer Cell, Rakesh Kumar and colleagues report the surprising finding that two cytoskeletal proteins, p21-activated kinase 1 (PAK1) and the dynein light chain 1 (DLC1), interact to promote the survival and tumorigenic potential of breast cancer cells.

Kumar and colleagues began their studies by looking for new substrates of PAKs. PAKs phosphorylate RHO GTPases to control cytoskeletal organization, and also phosphorylate and inactivate the pro-apoptotic protein $\mathrm{BAD}$ to promote survival. In a yeast two-hybrid screen of a mammarygland cDNA library, they found that PAK1 interacts directly with, and also phosphorylates, DLC1 - a component of the dynein motor complex. DLC1 not only regulates the microtubule-dependent motor function of dynein, but has also been shown to bind and inhibit activity of the pro-apoptotic protein BimL.

So what happens when these two pro-survival signalling proteins get together? Kumar and colleagues expressed normal and mutant forms of the proteins in a breast cancer cell line, and showed that the interaction between PAK1 and DLC1 is required for cell-cycle progression and survival. Cells that overexpressed either PAK1 or DLC1 were able to undergo anchoragein d e p e n d e n $\mathrm{t}$ growth, indicating a malignant phenotype, and also led to oestrogen-independent tumour growth when transplanted into nude mice, unlike control cells. A DLC mutant that lacked the PAK1 phosphorylation site did not show tumorigenic potential in mice.
Therefore, PAK1 phosphorylation of DLC1 seems to be required for cell survival and tumour formation. Furthermore, DLC1 levels were increased in $90 \%$ of the human breast tumour samples that the authors analysed.

How does PAK1 activation of DLC1 inhibit apoptosis and promote tumorigenesis? The authors propose a model whereby DLC1 sequesters BimL to the microtubules. Following pro-apoptotic signals, DLC1-BimL dimers are released and are free to inhibit BCL2, leading to cell death. When cells are exposed to growth factors or other survival signals, PAK1 becomes activated, leading to phosphorylation of DLC1 and BimL. This prevents the ability of the DLC1-BimL dimer to interact with and inhibit BCL2, leading to cell survival. So, increased levels of either PAK1 or DLC1 could promote cell survival and tumorigenesis. Further experiments are required to support this model and to investigate the role of these proteins in other tumour types.

\section{Kristine Novak}

(2) References and links ORIGINAL RESEARCH PAPER Vadlamudi, R. K. et al. Dynein light chain 1, a p21-activated kinase 1-interacting substrate, promotes tumorigenesis. Cancer Cell 5, 575-585 (2004) WEB SITE

Rakesh Kumar's lab:

http://gsbs.gs.uth.tmc.edu/tutorial/kumar.html

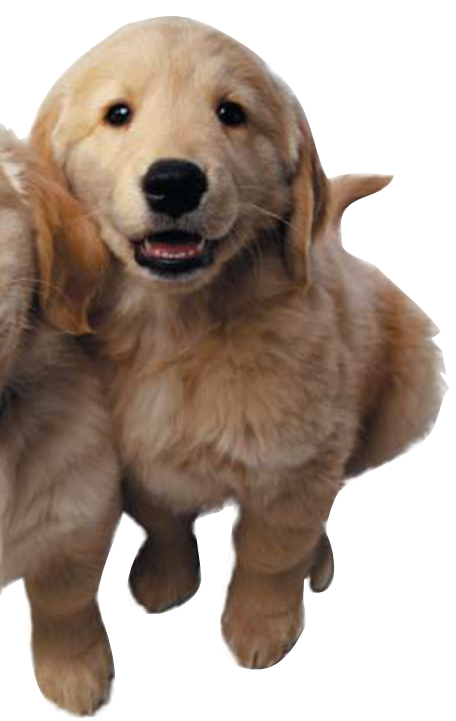

\section{IN BRIEF}

BREAST CANCER

$\mathrm{NF}-\kappa \mathrm{B}$ activation in human breast cancer specimens and its role in cell proliferation and apoptosis.

Biswas, D.K. et al. Proc. Natl Acad. Sci. USA 101, 10137-10142 (2004)

Oestrogen-receptor-negative breast tumours represent a significant therapeutic hurdle because of a lack of effective molecular targets. Biswas et al. show that tumours that are oestrogen-receptornegative but ERBB2-positive express increased levels of activated NF- $\kappa$ B. Suppression of NF- $\kappa$ B induced apoptosis in proliferating breast carcinoma cells, indicating that it might be a therapeutic target in these tumours.

\section{TUMORIGENESIS}

\section{SMYD3 encodes a histone methyltransferase involved} in the proliferation of cancer cells.

Hamamoto, R. et al. Nature Cell Biol. 4 July 2004 (doi:10.1038/ncb1151)

Methylation of DNA and its associated histones is important for the activation and repression of transcription. Hamamoto et al. show that SMYD3 is overexpressed in most colorectal and liver tumours. A specific domain in SMYD3 was found to methylate histone $\mathrm{H} 3$ and - as part of a complex of proteins that includes RNA polymerase II - SMYD3 regulates the transcription of several important cell-cycle control genes. The deregulated expression of SMYD3 in these tumours might be a key factor in their development.

\section{THERAPEUTICS}

\section{Histone deacetylase inhibitors specifically kill} nonproliferating tumour cells.

Burgess, A. et al. Oncogene 5 July 2004 (doi:10.1038/sj.onc.1207893)

Burgess and colleagues show that histone deacetylase inhibitors (HDIs) kill non-proliferating tumour cells, but not normal cells. The mechanism of cytotoxicity in non-proliferating cells involves the activation of the cyclin-dependent kinase inhibitor WAF1 the same pathway that is activated by HDIs in proliferating cells — but non-proliferating cells take longer to activate the apoptosis machinery. Therefore, HDIs might be useful in the treatment of slowly proliferating tumours.

\section{GENE EXPRESSION}

Inhibition of fatty acid synthase (FAS) suppresses HER2/neu (erbB-2) oncogene overexpression in cancer cells.

Menendez, J. A. et al. Proc. Natl Acad. Sci. USA 2 July 2004 (doi:10.1073/pnas.0403390101)

Levels of fatty-acid synthase (FAS) — an essential enzyme in the conversion of dietary carbohydrates to fatty acids - increase during breast cancer development, and hyperactivity of FAS is associated with aggressive disease. Javier Menendez et al. show that increased FAS activity in breast and ovarian cancer cell lines has an active role in malignant transformation by regulating key oncogenes, such as ERBB2. 